dat gaat buiten de kerf, litt. cela dépasse l'entaille, cela passe toutes les bornes ; op de kerfstok zetten, litt. indiquer sur la taille, porter en compte ; de kerfstok afdoen, effacer la taille, payer ce qu'on doit ; de kerfstok is ool, la taille est pleine, la mesure est comble ; hij heeft viel op zye kerfstok, il a pour beaucoup sur sa taille, il n'a pas la conscience nette. - En français, je ne connais qu'une expression qui rappelle cet usage : "prendre à la coche ", c'est-à-dire prendre à crédit.

Dans une autre direction, M. Sauvage, l'éminent connaisseur des choses normandes, écrivait à Dom Berthet le 2 juin 1950 pour lui communiquer un passage de la conclusion de son Histoire de l'abbaye de Troarn, allégué par MM. Blache et Latouche, et dont il tenait à lui communiquer le texte original. Il s'agit de l'article de Dom Berthet intitulé Abbayes et exploitations : l'exemple de Saint-Claude ${ }^{1}$.

Nous ne voulions d'abord découvrir dans les Archives de Troarn que des renseignements sur l'histoire des relations économiques et sociales dans un quartier de la Vallée d'Auge. L'insignifiance et la rareté des documents "spirituels " que l'on a conservés nous disposaient à cette attitude. L'évidence, pourtant, nous a instruit. Les actes mêmes de l'abbaye de Troarn les plus pénétrés de l'esprit utilitaire, décèlent chez les moines et chez leurs clients d'autres préoccupations que les seules préoccupations du siècle. Les actes les plus "économiques" de l'abbaye sont marqués du caractère religieux. Le rôle de Saint-Martin de Troarn comme banque, comme maison de crédit, fut réel sans aucun doute - mais subsidiaire : l'abbaye se livra, pour son profit immédiat d'abord, et pour le profit, par contrecoup, des populations rurales, à des entreprises de finance ; mais la nature de ces entreprises fut troublée, faussée, si l'on veut, par le sentiment religieux. Au xi ${ }^{\mathbf{e}}$, au $\mathrm{XII}^{\mathrm{e}}$, au $\mathrm{XIII}^{\mathrm{e}}$ siècle, jusqu'au développement de l'autorité centrale et à l'ingérence de ses officiers, à l'époque du rôle économique réel et variable des monastères, les moines de Troarn unissaient les soucis spirituels aux temporels. Jusqu'à la fin il demeura toujours quelque chose de cette union primordiale. - En somme, nous estimons, simplement, qu'il ne convient pas d'oublier qu'une abbaye est une institution religieuse $2 . .$.

Et M. Sauvage d'ajouter : " Cette longue citation, dont je m'excuse, découvre, je crois, mon point de vue plus nuancé que celui des auteurs qui m'ont fait l'honneur de me lire, et, je le crois aussi, tout proche du vôtre. - LUCIEN FEbVRE.

\title{
LE MOT D'UNE ÉNIGME ?
}

J'ai été, comme bien d'autres - André Allix pour ne citer que lui assez perplexe quand il m'a fallu rendre compte, ici, du second volume de La France économique et humaine d'Albert Demangeon ${ }^{3}$. Or, voici que Jean Gottmann m'écrit une lettre d'où j'extrais le passage suivant :

Vous avez, m'écrit-il, dans votre compte rendu, mis le doigt très justement sur les inégalités du plan - et, en particulier, sur la dispersion de l'étude de la circulation. Ayant vécu auprès de Demangeon les années où il écrivait ce livre, je crois

1. Annales (E. S. C.), janvier-mars 1950 , p. 68 . Voici le texte de M. Blache : c On peut répéter avec M. Latouche que.les Chartreux..., lorsqu'ils sontvenus chercher dans les montagnes du Vercors une retraite presque inaccessible, n'avaient pas l'intention de coloniser le pays et ouligner avec M. Sauvage qu'une abbaye est une construction religieuse. "

2. Histoire et débeloppement économique d'un monastère normand au moyen dge. L'abbaye de Saint-Martin de Troarn au diocese de Bayeux, des origines au XVI ${ }^{\circ}$ siècle, Caen, 1911, in-4º, p. 282-283.

3. Voir mon compte rendu dans les Annales, t. IV (1949), p. 64 et suiv. 
pouvoir vous assurer que les hésitations de la rédaction traduisent plus que de la hâte et de l'inachevé ; elles reproduisent des hésitations de la pensée, le sentiment qui poussait d'un rôle de la circulation en géographie humaine bien plus important qu'on n'avait eu l'habitude de le dire. Vers cette époque, dans ses Cours, Demangeon avait commencé de mettre la circulation en tête de sa Géographie économique - avant l'agriculture et l'industrie. Le plan classique en était révolutionné. La mort ne lui laissa pas le temps de donner une forme définitive à l'ordre nouveau dont il sentait le besoin. Le "désarroi "du plan constitue donc une indication d'évolution, une étape dans la méthode... J'en ai discuté plusieurs fois avec lui - et j'ai pensé que ce petit " témoignage " pourrait vous intéresser.

Il intéressera non seulement ma vieille et fidèle amitié pour Demangeon - mais tous les lecteurs de son livre posthume. L'interprétation de Jean Gottmann rend compte, en effet, de ce qu'il y avait d'étrange dans l'espèce de "tournoiement " d'un homme qui, dans tous ses livres et dans tous ses articles, a toujours donnédes modèles de plans lucides, simples et d'une excellence classique. Je dirai volontiers que la lecture de la lettre dont je tiens à donner un extrait à nos lecteurs m'a soulagé d'un poids. J'étais troublé. Comme on l'est toujours quand on ne comprend pas bien. Je ne le suis plus. Et je ne puis que reconnaître Demangeon, "notre Demangeon ", dans ce souci, à la fin de sa vie, de se renouveler encore et toujours, de repenser ses idées et de chercher à serrer do plus près en plus près la réalité. C'est d'un bel exemple - et c'était bien de lui. - L. F.

\section{MEA CULPA}

Un lecteur neuchatelois, M. Mermod, m'écrit pour me signaler que, dans les brèves notes que j'ai mises à la suite de Métier d'historien, je signale que " pas une seule fois sauf erreur, dans tout le livre, le mot d'éyolution n'est prononcé ». Et mon correspondant de me demander " pourquoi j'ai l'air de reculer devant ce mot qui lui paraît, en de telles matières, simplement synonyme de développement ". - Après quoi il me fait observer que je fais erreur matériellement, car il relève (notamment p. 9, 73, 92, 94) dans l'ouvrage de Marc Bloch le mot que je pensais absent.

Il a raison. Comment ai-je commis cette erreur ? Simplement parce que j'ai rédigé ma note sur le manuscrit, ou plutôt d'après le manuscrit - et chacun sait qu'on ne tient jamais un manuscrit sous les yeux (quand on n'en est pas soi-même l'auteur) comme on tient un imprimé. - Je m'excuse simplement d'avoir parlé trop vite. Mal défendu par un "sauf erreur » derrière lequel je ne vais pas m'abriter.

Quant à la question de fond? Ce n'est pas moi qui recule devant le mot "évolution ". Si j'ai, après une lecture trop rapide sur ce point, rédigé la note qu'on sait, c'est en me référant à d'anciennes conversations avec Marc Bloch - qui ne tenait pas "évolution" pour synonyme, simplement, de "développement". Et qui était soucieux de bannir de l'histoire (comme moi-même) toutes ces grandes machines, toutes ces "forces " nées de notro cerveau et dont nous faisons sans plus de cérémonie des réalités causales. - L. F. 